Benchmarks

\title{
Method for improved Illumina sequencing library preparation using NuGEN Ovation RNA-Seq System
}

Steven R. Head ${ }^{1}$, H. Kiyomi Komori ${ }^{2}$, G. Traver Hart ${ }^{2}$, John Shimashita ${ }^{1}$, Lana Schaffer ${ }^{1}$, Daniel R. Salomon ${ }^{2}$, and Phillip T. Ordoukhanian ${ }^{1}$

${ }^{I}$ Next Generation Sequencing Core, The Scripps Research Institute, La Jolla, CA, USA and ${ }^{2}$ Department of Molecular \& Experimental Medicine, The Scripps Research Institute, La Jolla, CA, USA

BioTechniques 50:177-181 (March 2011) doi 10.2144/000113613

Keywords: RNA-seq; Next-generation sequencing; deep sequencing; gene expression; cDNA library

Supplementary material for this article is available at www.BioTechniques.com/article/113613.

In this study, we tested the NuGEN Ovation RNA-Seq System for library preparation followed by next-generation sequencing on an Illumina GAIIx. The cDNA product of the NuGEN kit may have significant amounts of ssDNA with hairpin structures that are generated during the amplification process. These structures interfere with efficient downstream end repair, A-tailing, and adapter ligation, all standard steps in post-amplification sequencing library construction. We were able to increase the efficiency of sequencing library yields 4- to 6-fold or greater by treatment of NuGENamplified cDNA product with the single-strand endonuclease S1. These results suggest that this treatment effectively cleaves hairpin structures generated during amplification that are resistant to the standard enzyme cocktails used for the end-repair step.

Next-generation sequencing (NGS) technology has significantly altered the economics of genomics and opened up new avenues of research such as wholegenome and exome sequencing (1), RNA-seq $(2,3)$, ChIP-seq, and small RNA-seq (4) that were previously costprohibitive. New methods for more efficient and lower cost preparation of sequencing libraries have been developed (5) and new commercial kits provide reagents and consumables for specific NGS applications.

NuGEN (San Carlos, CA, USA) recently introduced a new protocol for RNA-seq to simplify library preparation starting from small amounts of total RNA without preselection of mRNA or other steps to reduce rRNA contamination. The NuGEN kit uses a single primer isothermal amplification (SPIA) method to amplify RNA target into cDNA. This cDNA can then be brought into standard Illumina (San Diego, CA, USA) library preparation protocols that involve end repair, A-tailing, and ligation of selected sequencing adapters.

Initial testing of NuGEN-amplified cDNA for library preparation yielded lower-than-expected amounts of properly ligated product when compared with DNA libraries prepared with our standard protocols, where we routinely begin with 100 ng dsDNA. We hypothesized that the end-repair steps critical to the Illumina protocol may not be as efficient for the NuGEN-amplified cDNA due to hairpin structures formed at the cDNA ends. Since the end-repair enzymes include single-strand exonuclease and polymerase functions, hairpin structures would be resistant to blunt-end conversion by these enzymes. To test this hypothesis, we treated NuGEN-amplified cDNA with the single-strand-specific endonuclease $S 1$ (Promega, Madison, WI, USA; $50 \mathrm{U} / \mu \mathrm{L}$ for $30 \mathrm{~min}$ at room temperature). This treatment should cleave any hairpins created during the NuGEN protocol allowing them to convert to blunt-end cDNA using the standard end-repair cocktail in the Illumina protocol.

Initially, a series of six total RNA samples were extracted from human CD4 $+\mathrm{T}$ cells before and after activation with anti-CD3/anti-CD28 beads (Invitrogen, Carlsbad, CA, USA), a well-established model for the immune response (6). Total RNA was prepared using standard TRIzol (Invitrogen) methods for four of the samples and AllPrep (Qiagen, Valencia, CA, USA) RNA purification for two of the samples. After confirming RNA quantity and integrity [Nanodrop (Wilmington, DE, USA) and Agilent Bioanalyzer (Santa Clara, CA, USA) analysis, respectively; see BioAnalyzer traces in the Supplementary Materials], $100 \mathrm{ng}$ each sample was converted to cDNA using the NuGEN protocol. Then 100 ng NuGEN-amplified cDNA from each sample was taken into a standard Illumina sequencing library preparation directly or after treatment with S1 endonuclease. We tested library products at two size ranges of $\sim 300 \mathrm{bp}$ and $750 \mathrm{bp}$ in length, corresponding to typical protocols for short- and long-read sequencing, respectively. The two products were isolated from agarose gels and PCR-amplified for 15 cycles using Phusion polymerase (Finnzymes, Vantaa, Finland). PCR products were then analyzed on a $2 \%$ agarose gel (Figure $1 \mathrm{~A}$ ). The longer library products are close to the limit of what can efficiently form clusters for sequencing on Illumina flowcells but would be suitable for longer read technologies.

It is clear from the gel results (Figure 1A) that S1 endonuclease treatment of the cDNA before proceeding with library preparation significantly enhanced the yield for both products. In fact, the larger 750-bp products are not detectable at all in the untreated samples but are clearly visible in the S1-treated samples. Quantitative analysis of the gel image confirmed these conclusions (Figure 1B).

In a second experimental series, we tested whether simply increasing the input amount of cDNA could improve the yields. A single sample was processed using two different input amounts of cDNA 
A

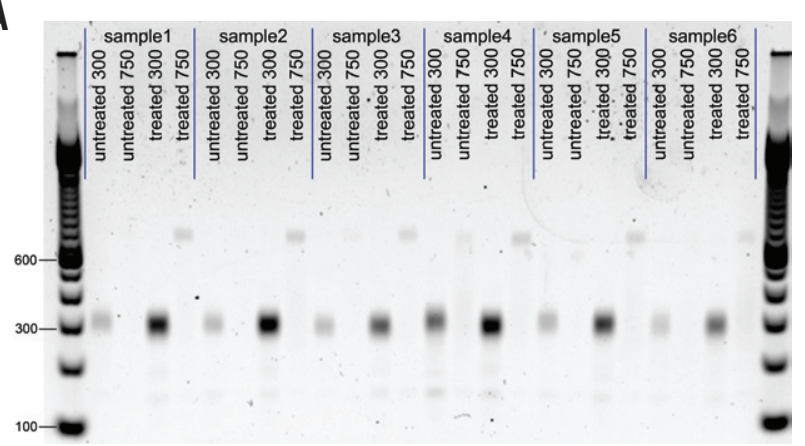

B

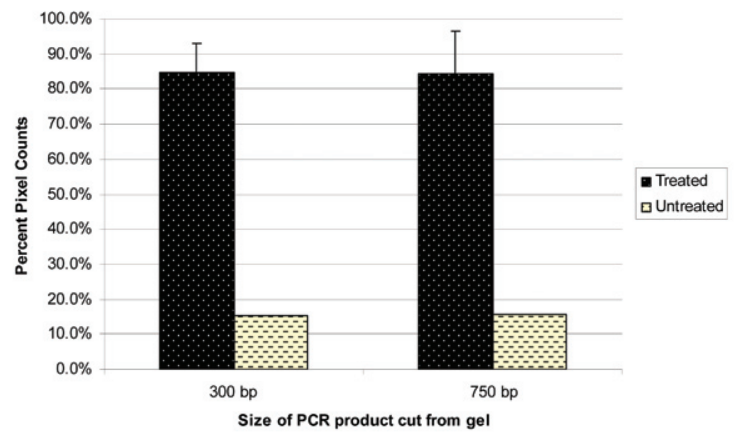

Figure 1. (A) Gel images of PCR-amplified library preparations of six CD4 T cell samples. In order to assess the impact of S1 treatment, we prepared cDNA libraries of two different sizes, $300 \mathrm{bp}$ and $750 \mathrm{bp}$. The sizing ladder run in the outside lanes is a 100-bp ladder with the 100-bp, 300-bp, and 600-bp markers indicated. All samples were prepared using the NuGEN Ovation RNA-Seq system followed by end repair, A-tailing, and sequencing adaptor ligation and then 15 cycles of PCR. Untreated = no treatment with S1 endonuclease. Treated = pre-treatment of cDNA with S1 endonuclease prior to going forward with the rest of the library preparation protocol as already described above. (B) Quantitative analysis of 300-and 750-bp RNA-seq libraries prepared with and without S1 nuclease treatment. Mean and SD of relative percentage yields based on the total pixel counts for treated and untreated sample pairs are shown as quantitated using the Bio-Rad Gel Doc software. One SD value is shown for each product size since the sum of treated and untreated percentages equals $100 \%$ for each pair.

A

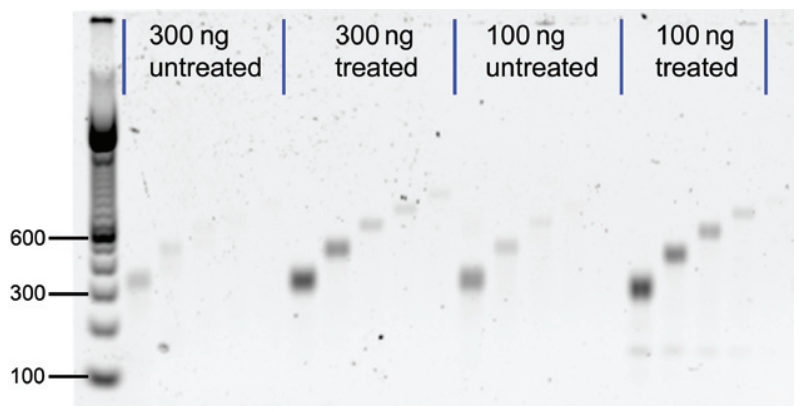

B

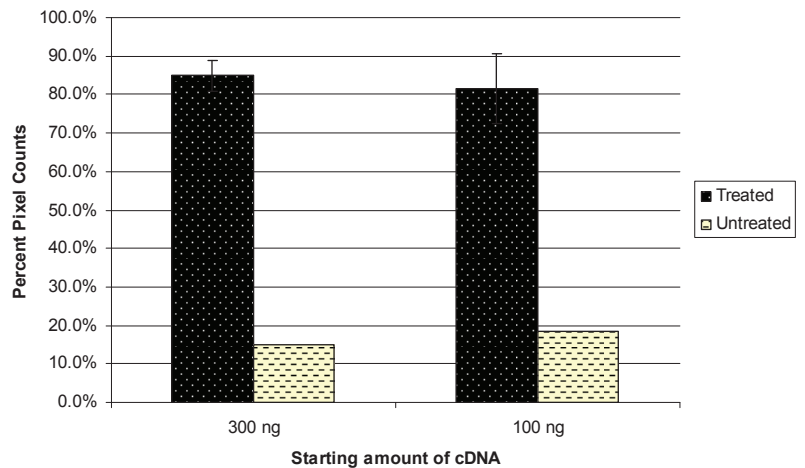

Figure 2. (A) Gel images of PCR-amplified library preparations starting with either $100 \mathrm{ng}$ or $300 \mathrm{ng}$ cDNA. cDNA was either treated with S1 endonuclease (treated) or not (untreated) prior to our standard Illumina library preparation protocol. The sizing ladder run in the outside lanes is a 100-bp ladder with the 100-bp, 300-bp, and 600-bp markers indicated. Bands were excised from a 2\% agarose gel at 300-, 400-, 500-, 600-, and 750-bp sizes and then amplified by 15 cycles of PCR. The $2 \%$ agarose gel of the corresponding PCR products is shown. (B) Mean and SD of relative percentage yields based on the total pixel counts for treated and untreated sample sets starting with $300 \mathrm{ng}$ and $100 \mathrm{ng}$ input cDNA are shown as quantitated using the Bio-Rad Gel Doc software (Hercules, CA, USA). One sD value is shown for each product size, since the sum of treated and untreated percentages equals $100 \%$ for each pair.

in the library preparation. Aliquots of $100 \mathrm{ng}$ and $300 \mathrm{ng}$ were either S1-treated or left untreated before continuing the protocol to end-repair, A-tailing, and adapter ligation. These four libraries were run on $2 \%$ agarose gels and bands were cut out at 300, 400, 500, 600, and $750 \mathrm{bp}$ followed by 15 cycles of PCR. After PCR, the size-selected library products were run on a second $2 \%$ agarose gel (Figure 2A) and quantified (Figure 2B). Again, results show the improved yield after $S 1$ treatment for both input amounts. In this case we found somewhat better yields

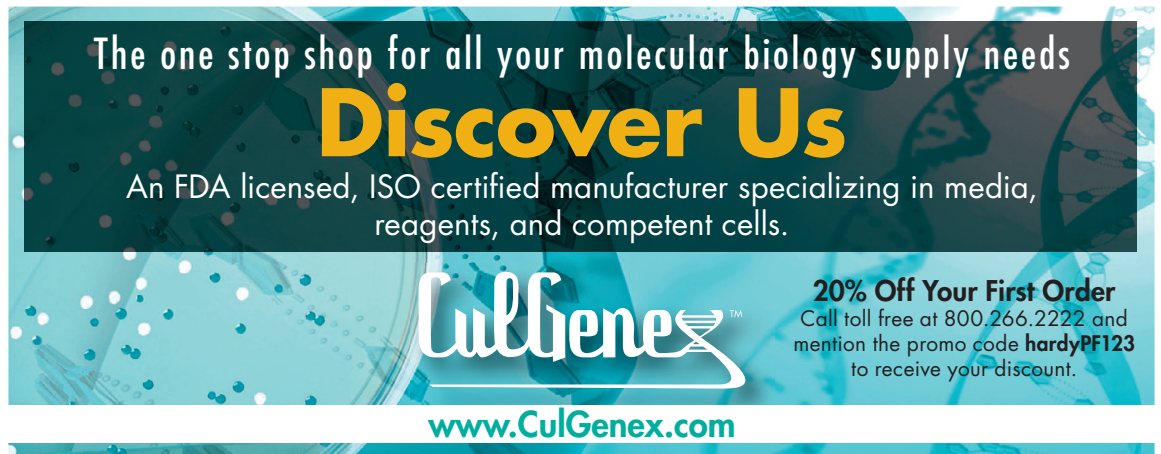

with $100 \mathrm{ng}$ relative to $300 \mathrm{ng}$ input cDNA. It is probable that these better yields were the result of reaction conditions more optimal for starting with a smaller amount of cDNA. We performed an additional experiment using $1 \mu \mathrm{g}$ cDNA and library preparation reagent concentrations optimized for this scale of reaction, and observed increased yields for S1-treated cDNA (4-20-fold) while reducing the total number of PCR cycles (6-8) (see Supplementary Figures S6 and S7). Thus, the S1 treatment is beneficial for improving library yields under a variety of optimized library preparation conditions.

All the S1-treated 300-bp libraries shown in Figure 1A were then sequenced on an Illumina GAIIx to generate pairedend reads of $60 \mathrm{bp}$ each. Resulting data were aligned using Pipeline1.5_ 
Table 1. Alignment statistics for RNA-seq.

\begin{tabular}{|c|c|c|c|c|c|c|}
\hline Sample & Description & $\begin{array}{l}\text { RNA extraction } \\
\text { method }\end{array}$ & $\begin{array}{c}\text { Total number of } \\
\text { reads }\end{array}$ & mRNA & Mitochondrial & IRNA \\
\hline 1 & $\begin{array}{l}\text { Donor } 1 \text {; unactivated } \\
\text { CD4 cells }\end{array}$ & TRIzol (Invitrogen) & $30,440,976$ & $41.6 \%$ & $12.1 \%$ & $9.6 \%$ \\
\hline 2 & $\begin{array}{l}\text { Donor } 1 ; 48 \mathrm{~h} \text { activated } \\
\text { CD4 cells }\end{array}$ & TRIzol (Invitrogen) & $37,765,048$ & $34.1 \%$ & $19.6 \%$ & $16.4 \%$ \\
\hline 3 & $\begin{array}{l}\text { Donor } 2 \text {; unactivated } \\
\text { CD4 cells }\end{array}$ & TRIzol (Invitrogen) & $30,772,248$ & $47.9 \%$ & $9.1 \%$ & $8.1 \%$ \\
\hline 4 & $\begin{array}{c}\text { Donor } 2 ; 48 \mathrm{~h} \text { activated } \\
\text { CD4 cells }\end{array}$ & TRIzol (Invitrogen) & $35,383,114$ & $34.9 \%$ & $19.8 \%$ & $14.5 \%$ \\
\hline 5 & $\begin{array}{c}\text { Donor } 3 \text {; unactivated } \\
\text { CD4 cells }\end{array}$ & AllPrep (Qiagen) & $31,556,534$ & $19.5 \%$ & $12.1 \%$ & $35.3 \%$ \\
\hline 6 & $\begin{array}{c}\text { Donor } 3 ; 48 \mathrm{~h} \text { activated } \\
\text { CD4 cells }\end{array}$ & AlIPrep (Qiagen) & $37,818,452$ & $20.6 \%$ & $11.2 \%$ & $36.3 \%$ \\
\hline
\end{tabular}

We determined the total reads and percent alignments to mRNA (including splice junctions), mitochondrial and rRNA sequences using Pipeline1.5_CASAVA1.0 (Illumina) for all six samples. Each sample was run in a single lane. All the paired-end reads were split into single 60 -bp reads and then alignment was done with a filter set to $<3$ errors/read to the human genome as represented in HG18 (http://genome.ucsc.edu/cgi-bin/hgTracks?hgsid=185275249).

CASAVA1.0 (Illumina; Table 1). It is interesting to note that RNA extraction for samples 5 and 6 , representing resting and activated CD4 T cells from Donor 3, were done with the AllPrep RNA purification kit (Qiagen) and demonstrated significantly lower yields of unique matches for mRNA and correspondingly higher ribosomal RNA (rRNA) matches relative to RNA extracted using the TRIzol method. These results suggest that RNA extraction methods need careful evaluation for their impact on the RNA-seq data. The TRIzol method is performed primarily in solution phase using organic extraction, ethanol precipitation, and washes, whereas the AllPrep method purifies the RNA through a series of column separation steps. It is not clear how these differences would affect the final distribution of RNA-seq reads and due to the small sample size we hesitate to draw conclusions beyond our observations. All RNA samples were of similar high quality, with RIN scores of $>9$ (see Supplementary Material). The alignment statistics from libraries prepared using TRIzol-extracted RNA are $\sim 40 \%$.

Finally, in order to compare RNA-seq data from S1-treated and untreated libraries, we tested RNA from Donor 1 CD4+ memory and naive T cells (extracted using the AllPrep method). cDNA was prepared and subsequently either S1-treated or left untreated before continuing the protocol to end-repair, A-tailing, and adapter ligation. Each library was loaded into a separate lane of a single-read flow-cell and sequenced 40 bases. For all samples, the proportions of mRNA and mitochondrial reads were similar to Donor 3, also extracted using the AllPrep protocol. However the percent of rRNA reads was substantially lower in the memory and naive cell libraries and not influenced by S1 treatment (Supplementary Table S1). The reason for this difference in $\mathrm{rRNA}$ reads is not clear but may reflect an improvement in kit reagents by NuGEN during the time period between the processing of the first six samples and then the memory and naive samples $\sim 14$ months later.

Correlations between gene expression values as measured by reads per kilobase per million reads (RPKM) show consistent relative gene expression for both memory and naive cells in S1-treated and untreated samples (Spearman's rank correlation coefficient, >0.98; Supplementary Figures S3 and S4). Analysis of differential expression between memory and naive cells in the S1-treated and untreated sets also shows very high correlation (Pearson's correlation coefficient $\sim 0.82$ across $>9000$ genes with RPKM >1 for all four samples; Supplementary Figure S5). Finally, the percent of duplicate reads was analyzed for the four samples with values of $49 \%$ and $57 \%$ for the 51 -treated and untreated memory cells, respectively, and $42 \%$ and $50 \%$ for the S1-treated and untreated naive cells, respectively.

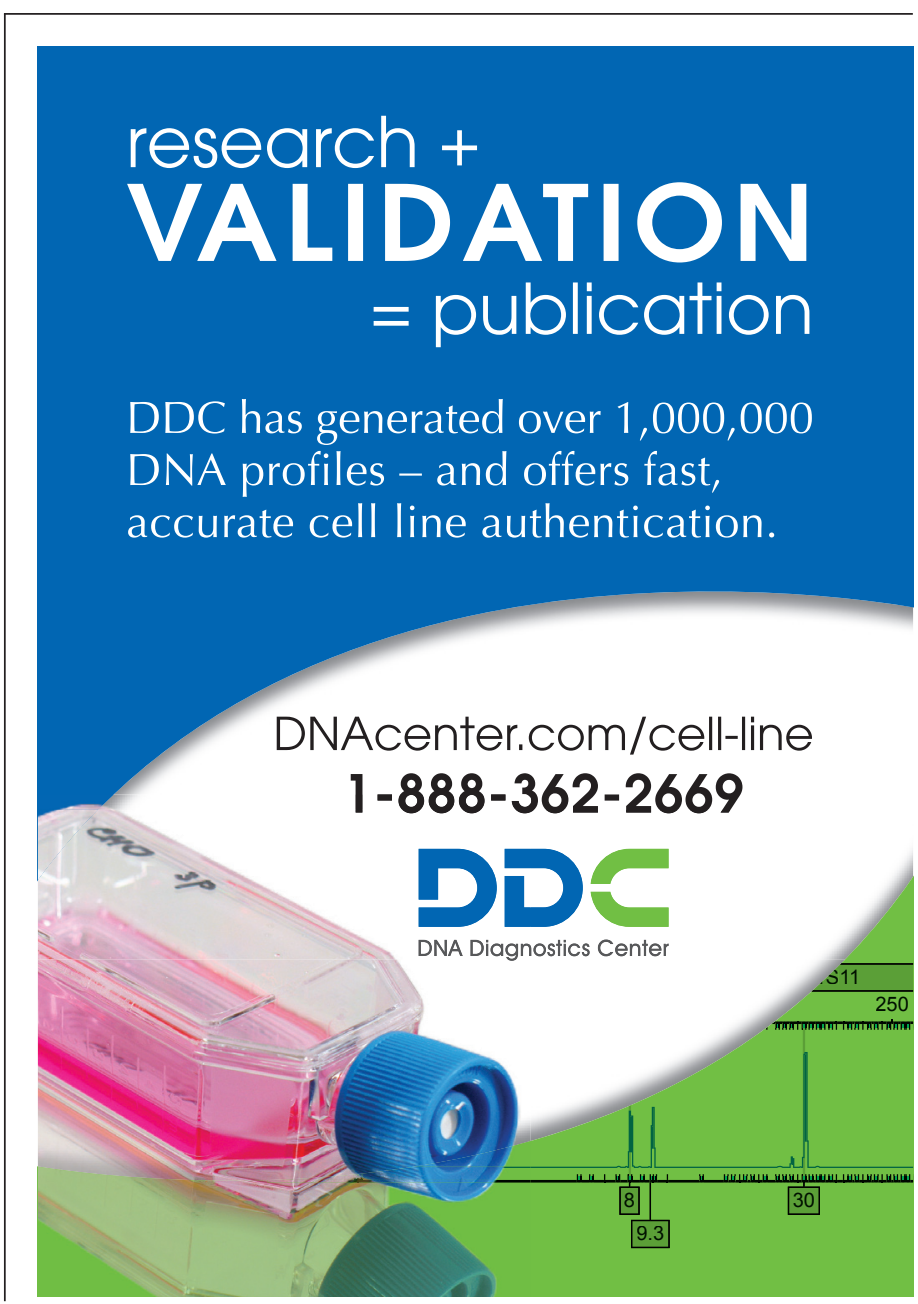


Overall, these results suggest some improvement in library quality resulting from the $S 1$ treatment.

In conclusion, we have successfully performed RNA-seq using the NuGEN Ovation RNA-Seq System on multiple samples of total RNA prepared from human CD4+ T cells. The results demonstrate that the addition of the pretreatment step with S1 endonuclease improves the yields of sequencing libraries by 4-6-fold or greater without reducing the quality of the library. Indeed, the advantages of improving yield include a reduction in the number of PCR cycles required for the protocol, thus potentially reducing PCR bias and duplicate reads, and thereby improving the complexity in the sequencing library. We believe that the addition of the S1 treatment is particularly relevant for preparing sequencing libraries from longer cDNA products where lower yields are more problematic. Thus the addition of the S1 endonuclease step into the current NuGEN protocol is a technically simple and low-cost way to maximize yields of high-quality cDNA libraries produced for RNA-seq.

\section{Acknowledgments}

This research was supported by funds from the National Institutes of Health [NIH; grant nos. U19 A1063603-06 (to D.R.S., S.R.H., J.S., and L.S.) and T32DK007022-30 (to HKK)], the Molly Baber Research Fund, and the Verna Harrah Research Fund supporting the Salomon laboratory. The NuGEN Ovation kits and additional support for deepsequencing were provided in the form of reagents as an unrestricted research grant from NuGEN. This paper is subject to the NIH Public Access Policy.

\section{Competing interests}

The authors declare no competing interests. There were no consulting fees, existing contracts, stocks or options, intellectual property arrangements, or any other form of conflict of interest issues between the Scripps researchers and NuGEN. No $\mathrm{NuGEN}$ employee had any role in the experimental designs or the data interpretation and manuscript preparation.

\section{References}

1. Archibald, A.L., L. Bolund, C. Churcher, M. Fredholm, M.A. Groenen, B. Harlizius, K.T. Lee, D. Milan, et al. 2010. Pig genome sequence-analysis and publication strategy. BMC Genomics 11:438.

2. Hosseini, P., A. Tremblay, B.F. Matthews, and N.W. Alkharouf. 2010. An efficient annotation and gene-expression derivation tool for Illumina Solexa datasets. BMC Res Notes. 3:183.

3. Martí, E., L. Pantano, M. Bañez-Coronel, F. Llorens, E. Miñones-Moyano, S. Porta, L. Sumoy, I. Ferrer, et al. 2010. A myriad of miRNA variants in control and Huntington's disease brain regions detected by massively parallel sequencing. Nucleic Acids Res. 38:7219-7235.

4. Hawkins, R.D., G.C. Hon, L.K. Lee, Q. Ngo, R. Lister, M. Pelizzola, L.E. Edsall, S. Kuan, et al. 2010. Distinct epigenomic landscapes of pluripotent and lineagecommitted human cells. Cell Stem Cell 6:479-491.

5. Quail M.A., I. Kozarewa, F. Smith, A. Scally, P.J. Stephens, R. Durbin, H. Swerdlow, and D.J. Turner. 2008. A large genome center's improvements to the Illumina sequencing system. Nat. Methods. 5:1005-1010.

6. Trickett, A. and Y.L. Kwan. 2003. T cell stimulation and expansion using anti-CD3/ CD28 beads. J. Immunol. Methods 275:251255.

Received 18 October 2010; accepted 1 February 2011.

Address correspondence to Steven R. Head, Next Generation Sequencing Core, The Scripps Research Institute, 10550 N. Torrey Pines Road, La Jolla, CA, 92040, USA. e-mail: shead@scripps.edu

To purchase reprints of this article, contact: carmelitag@fosterprinting.com

\section{a mini centrifuge that holds Four PGR Strips!}

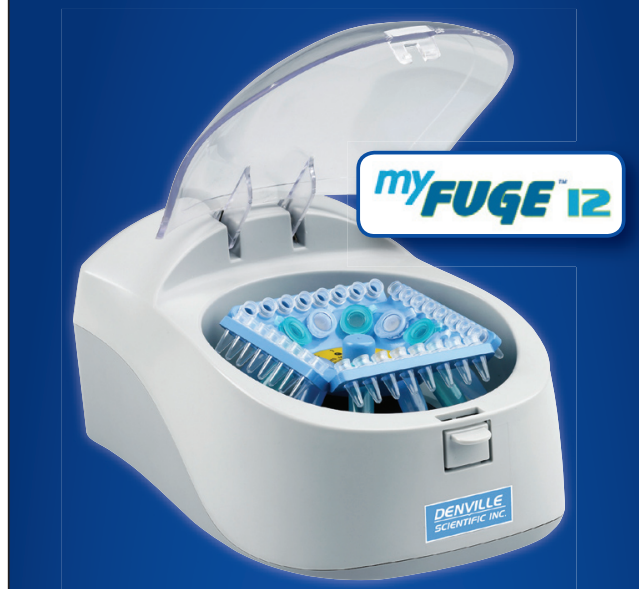

- Run 4 PCR Strips \& $12 \times 1.5 \mathrm{ml}$ tubes with the COMBI-Rotor

- 5,500 rpm/Near Silent Operation

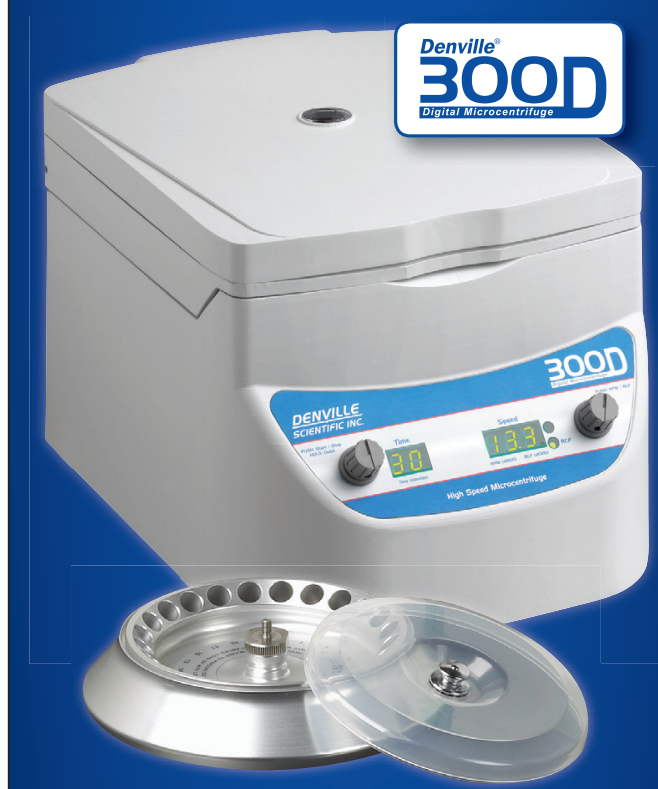

- Cold Room Safe

- 13,300 rpm/24 Place Rotor

- Small Footprint (10.5 x 12 x 10 in.)

Denville Scientific...

Your Source for Centrifuges

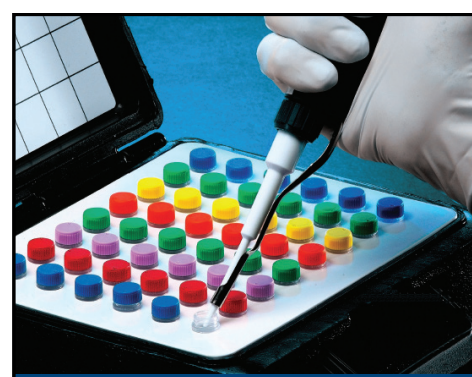

The Cooler Store FEATURING THE mand Enzyme storage Bench top coolers Aluminum blocks \& racks Check out our new,
easier-to-use website! BiOSMith Biosmith.com
1.800 .929 .7894

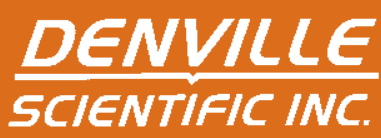

800.453.0385 | wuru.densci.com 